\title{
Ambulatory Assessment of Ankle and Foot Dynamics
}

\author{
H. Martin Schepers*, Student Member, IEEE, H. F. J. M. Koopman, and Peter H. Veltink, Senior Member, IEEE
}

\begin{abstract}
Ground reaction force (GRF) measurement is important in the analysis of human body movements. The main drawback of the existing measurement systems is the restriction to a laboratory environment. This paper proposes an ambulatory system for assessing the dynamics of ankle and foot, which integrates the measurement of the GRF with the measurement of human body movement. The GRF and the center of pressure (CoP) are measured using two six-degrees-of-freedom force sensors mounted beneath the shoe. The movement of foot and lower leg is measured using three miniature inertial sensors, two rigidly attached to the shoe and one on the lower leg. The proposed system is validated using a force plate and an optical position measurement system as a reference. The results show good correspondence between both measurement systems, except for the ankle power estimation. The root mean square (RMS) difference of the magnitude of the GRF over 10 evaluated trials was $(0.012 \pm 0.001) \mathrm{N} / \mathrm{N}$ (mean \pm standard deviation), being $(1.1 \pm 0.1) \%$ of the maximal GRF magnitude. It should be noted that the forces, moments, and powers are normalized with respect to body weight. The CoP estimation using both methods shows good correspondence, as indicated by the RMS difference of $(5.1 \pm 0.7) \mathrm{mm}$, corresponding to $(1.7 \pm 0.3) \%$ of the length of the shoe. The RMS difference between the magnitudes of the heel position estimates was calculated as $(18 \pm 6) \mathrm{mm}$, being $(1.4 \pm 0.5) \%$ of the maximal magnitude. The ankle moment RMS difference was $(0.004 \pm 0.001) \mathrm{Nm} / \mathrm{N}$, being $(2.3 \pm 0.5) \%$ of the maximal magnitude. Finally, the RMS difference of the estimated power at the ankle was $(0.02 \pm 0.005) \mathrm{W} / \mathrm{N}$, being $(14 \pm 5) \%$ of the maximal power. This power difference is caused by an inaccurate estimation of the angular velocities using the optical reference measurement system, which is due to considering the foot as a single segment. The ambulatory system considers separate heel and forefoot segments, thus allowing an additional foot moment and power to be estimated. Based on the results of this research, it is concluded that the combination of the instrumented shoe and inertial sensing is a promising tool for the assessment of the dynamics of foot and ankle in an ambulatory setting.
\end{abstract}

Index Terms-Ambulatory measurement, center of pressure, dynamics, ground reaction force, inertial sensing.

\section{INTRODUCTION}

A NALYSIS of human body movement is commonly done in so-called 'gait laboratories'. In these laboratories, body movement is measured by a camera system using optical markers, the ground reaction force (GRF) using a force plate fixed in the floor, and the muscle activity using EMG. From the body movements and ground reaction forces, joint moments and powers can be estimated by applying inverse dynamics

Manuscript received July 6, 2006; revised October 15, 2006. This work was supported by the Dutch Ministry of Economic Affairs under the FreeMotion project. Asterisk indicates corresponding author.

*H. M. Schepers is with the Institute for Biomedical Technology (BMTI), University of Twente, P.O. Box 217, 7500 AE Enschede, The Netherlands (e-mail: h.m.schepers@ewi.utwente.nl)

H. F. J. M. Koopman and P. H. Veltink are with the Institute for Biomedical Technology (BMTI), University of Twente, 7500 AE Enschede, The Netherlands.

Digital Object Identifier 10.1109/TBME.2006.889769 methods [1]-[4]. The main disadvantage of such measurement system is that it is restricted to the gait laboratory. Therefore, research is required to find ways for performing these measurements outside the gait laboratory, for example in a doctor's practice, at the working place, or at home.

The measurement of the GRF using a force plate has several drawbacks. First, the subjects are required to place their feet completely on the force plates in order to perform a correct force measurement. This poses a restriction on the natural gait pattern. Second, only one or two steps can be measured during a trial, so many successive trials are usually required. Third, it is impossible to distinguish the GRF acting on each foot when standing with both feet on a single plate, as only the total GRF is registered. Finally, the force plate is fixed in the gait laboratory, which means the measurements cannot be performed in everyday life situations. Several research groups are attempting to overcome these limitations by constructing a "force plate" attached to the subject. A first attempt for an ambulatory measurement of the GRF was made by Kljajić and Krajnik [5], who described a system to measure the vertical component of the GRF and its distribution using force transducers beneath the shoe. The GRF has also been measured using pressure insoles [6]-[8]. However, like the system of Kljajić and Krajnik, these insoles only yielded the vertical component of the GRF. Therefore, additional knowledge was needed to estimate the shear components. Forner-Cordero et al. [8] solved this by using knowledge of body movements. Another solution was given by Savelberg and De Lange [9] who used pressure insoles in combination with an artificial neural network to achieve a relationship between pressure patterns and the shear component of the GRF. A miniature triaxial piezoelectric transducer measuring three orthogonal stress components inside a shoe was described by Razian and Pepper [10]. However, an independent and complete measurement of the GRF is preferred. An example was given by Roland et al. [11], who described the design and demonstration of a dynamometric horseshoe for measuring GRFs of horses during racing conditions. Chao and Yin [12] presented a novel shoe-shape structure, capable of mounting two six degree-of-freedom force sensors placed in the front part and rear part of that structure. This similar principle was proposed by Veltink et al. [13] using orthopaedic shoes equipped with six degrees of freedom force sensors (Fig. 1). In the design of Chao and Yin [12] the movement is constrained by a hinge, positioned between the front and rear sensor, while the design of Veltink $e t$ al. [13] allows distributed deformation of the shoe sole. An essential component still missing in this design is the measurement of the force sensor movement. In contrast to the force plate measurement, the force sensors follow the foot movement, which means the orientation of the force sensors has to be measured for an accurate estimation of the GRF.

Measuring body movement by a motion tracking system, using reflective markers attached to the body, offers accurate 


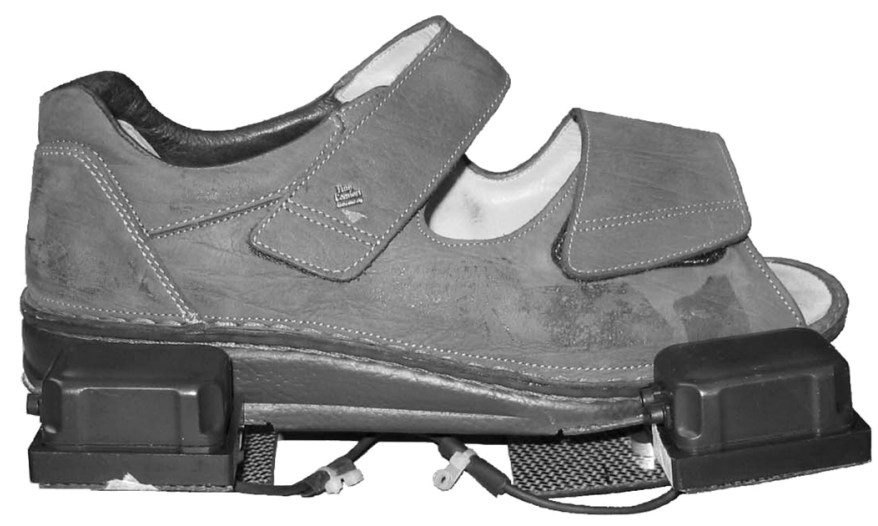

Fig. 1. Instrumented shoe with force transducers, and inertial sensors.

position tracking of body segments. However, this system has several drawbacks. First, the line of sight from camera to marker is often blocked by the subject, resulting in incomplete data. Second, these measurements are restricted to the laboratory environment also and, thus, cannot be performed in everyday life situations. An alternative is to use inertial sensors consisting of accelerometers and gyroscopes [14]-[17]. These sensors do not suffer from the above mentioned problems. However, obtaining the positions and orientations of a sensor by integration will introduce integration errors (drift). These errors can be avoided by using zero velocity updates [18], and knowledge of position and orientation [19], [20].

The objective of this study is to assess the ankle and foot dynamics by integrating the measurement of GRFs and body movement using an ambulatory system. The GRFs are measured using the instrumented shoes of Veltink et al. [13], whereas the movement of foot and ankle is measured using inertial sensors [19], [21], [22]. The system is validated using a force plate and an optical position measurement system as a reference.

\section{METHODS}

As mentioned in the introduction, this paper extends the work of Veltink et al. [13] by estimating the dynamics at the ankle and the foot. This section first describes the calculation of the moment and the power at the ankle and the foot. Subsequently, the calculation of the GRF and center of pressure (CoP) from the sensor signals is described, and the methods for estimation of the 3-D foot position and orientation are presented. Finally, the experimental methods are described.

\section{A. Moment and Power Calculation}

To obtain a full biomechanical analysis, it is desirable that the joint forces, moments and powers are estimated. In this research, a first attempt is made by estimating the force, moment, and power at the ankle. For calculation purposes, all vectors have to be expressed in the same coordinate system, being the global coordinate system. The origin and orientation of this global coordinate system $\Psi_{g}$ are renewed for each foot placement to coincide with the heel sensor coordinate system, when the heel is flat on the ground. Positive $x$ is in the direction of gait; positive $z$ is directed upward; and positive $y$ is perpendicular to the $x$ and

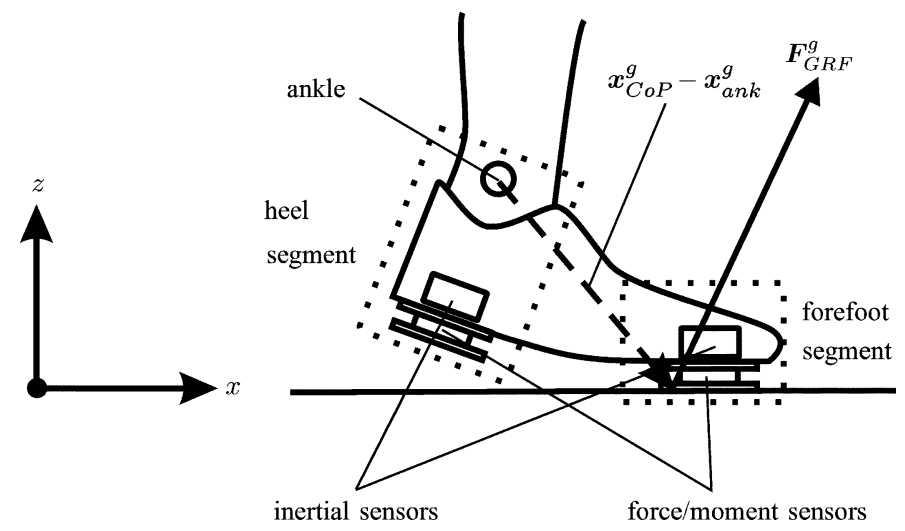

Fig. 2. Schematic drawing of the instrumented shoe during the stance phase.

$z$ direction such that the result is an orthogonal right-handed coordinate system. All measured signals are expressed in a sensor fixed coordinate system $\Psi_{s}$. This means that the measured signals have to be transformed to the global coordinate system $\Psi_{g}$.

A general change of coordinates between two coordinate systems $\left(\Psi_{i}\right.$ an $\left.\Psi_{j}\right)$ is denoted by

$$
\boldsymbol{H}_{i}^{j}=\left(\begin{array}{cc}
\boldsymbol{R}_{i}^{j} & \boldsymbol{p}_{i}^{j} \\
0_{3}^{T} & 1
\end{array}\right)
$$

where $\boldsymbol{R}_{i}^{j}$ is a rotation matrix representing the change of coordinates between frame $\Psi_{i}$ and $\Psi_{j}$ rotated with respect to each other, and $\boldsymbol{p}_{i}^{j}$ is a displacement vector representing the coordinates of the origin of frame $\Psi_{i}$ expressed in frame $\Psi_{j}$. The columns of the rotation matrix $\boldsymbol{R}_{i}^{j}$ are the coordinates of the unit axes of frame $\Psi_{i}$ expressed in frame $\Psi_{j}$

$$
\boldsymbol{R}_{i}^{j}=\left(\begin{array}{lll}
\boldsymbol{X}_{i}^{j} & \boldsymbol{Y}_{i}^{j} & \boldsymbol{Z}_{i}^{j}
\end{array}\right)
$$

This change of coordinates can for example be used to describe the position and orientation of a sensor, with attached frame $\Psi_{s}$, relative to a reference frame $\Psi_{g}$, by using $\boldsymbol{H}_{s}^{g}, \boldsymbol{R}_{s}^{g}$, and $\boldsymbol{p}_{s}^{g}$.

A schematic drawing of the instrumented shoe is shown in Fig. 2, where the foot has been divided in two segments. The force of each segment is measured by a force sensor, and the movement of each segment by an inertial sensor. The moment at the ankle joint $\boldsymbol{M}_{a n k}^{g}$ in global coordinates is calculated using the equations of motion [23], and the rotation matrix $\boldsymbol{R}_{s}^{g}$

$$
\begin{aligned}
\boldsymbol{M}_{a n k}^{g}=- & \left(\boldsymbol{x}_{\mathrm{CoP}}^{g}-\boldsymbol{x}_{a n k}^{g}\right) \times \boldsymbol{F}_{\mathrm{GRF}}^{g} \\
& +\left(\boldsymbol{x}_{f t}^{g}-\boldsymbol{x}_{a n k}^{g}\right) \times m_{f t} \boldsymbol{s}_{f t}^{g}+\boldsymbol{R}_{s}^{g} \frac{d}{d t}\left(\boldsymbol{I}_{f t} \boldsymbol{\omega}_{f t}^{s}\right)
\end{aligned}
$$

where the position of the ankle, the CoP, and the center of mass of the foot are given by $\boldsymbol{x}_{a n k}^{g}, \boldsymbol{x}_{\mathrm{CoP}}^{g}$, and $\boldsymbol{x}_{f t}^{g}$, respectively. The mass of the foot is given by $m_{f t}$, the moment of inertia of the foot by $I_{f t}$, the angular velocity of the foot by $\boldsymbol{\omega}_{f t}^{s}$, and the acceleration including gravity, expressed in global coordinates, by $\boldsymbol{s}_{f t}^{g}=\boldsymbol{a}_{f t}^{g}-\boldsymbol{g}^{g}$. Since the GRF contribution is considerably 
larger than the contribution of the inertial terms, the contribution of the inertial terms will be neglected [23]. This means (3) reduces to:

$$
\boldsymbol{M}_{\text {ank }}^{g}=-\left(\boldsymbol{x}_{\mathrm{CoP}}^{g}-\boldsymbol{x}_{\text {ank }}^{g}\right) \times \boldsymbol{F}_{\mathrm{GRF}}^{g} .
$$

The ankle power $P_{a n k}^{g}$ is calculated by the product of the ankle moment $\boldsymbol{M}_{\text {ank }}^{g}$ and the angular velocity at the ankle $\boldsymbol{\omega}_{\text {ank }}^{g}$, which is the difference between the angular velocity of the heel segment of the foot $\boldsymbol{\omega}_{h l}^{g}$ and the angular velocity of the lower $\operatorname{leg} \boldsymbol{\omega}_{\text {lleg }}^{g}$

$$
P_{a n k}^{g}=M_{a n k}^{g} \cdot \omega_{a n k}^{g}=M_{a n k}^{g} \cdot\left(\omega_{h l}^{g}-\omega_{l l e g}^{g}\right) .
$$

Since the foot has been modeled as two segments (Fig. 2), the heel and forefoot can move with respect to each other. This means a foot moment, and foot power are generated as well. The foot moment $\boldsymbol{M}_{f t}^{g}$, and power $\boldsymbol{P}_{f t}^{g}$ are calculated as

$$
\begin{aligned}
M_{f t}^{g} & =-\left(x_{C o P}^{g}-\boldsymbol{x}_{f t}^{g}\right) \times \boldsymbol{F}_{f f}^{g} . \\
P_{f t}^{g} & =\boldsymbol{M}_{f t}^{g} \cdot \boldsymbol{\omega}_{f t}^{g}=\boldsymbol{M}_{f t}^{g} \cdot\left(\boldsymbol{\omega}_{h l}^{g}-\boldsymbol{\omega}_{f f}^{g}\right)
\end{aligned}
$$

where $\boldsymbol{x}_{f t}^{g}$ denotes the position of an imaginary hinge point assumed to be present between the two foot segments, and $\boldsymbol{F}_{f f}^{g}$ the GRF measured by the forefoot sensor. The angular velocity of the foot is denoted by $\boldsymbol{\omega}_{f t}^{g}$, which is the difference between the angular velocities of the heel $\boldsymbol{\omega}_{h l}^{g}$, and the forefoot $\boldsymbol{\omega}_{f f}^{g}$.

\section{B. GRF and CoP Calculation}

As mentioned in the introduction, the GRF is measured by two six degree-of-freedom force sensors under the heel and the forefoot. The forces and moments measured by the sensors should be transformed to global coordinates, and combined. The transformation of the forces and moments measured by a force sensor with attached coordinate frame $\Psi_{s 1}$ to the global coordinate frame $\Psi_{g}$ is achieved using (1) and (2)

$$
\begin{aligned}
\boldsymbol{F}_{s 1}^{g} & =\boldsymbol{R}_{s 1}^{g} \boldsymbol{F}^{s 1} \\
\boldsymbol{M}_{s 1}^{g} & =\boldsymbol{R}_{s 1}^{g} \boldsymbol{M}^{s 1}+\boldsymbol{p}_{s 1}^{g} \times \boldsymbol{F}^{s 1} .
\end{aligned}
$$

The GRF $\boldsymbol{F}_{\mathrm{GRF}}^{g}$, and the moment $\boldsymbol{M}_{\mathrm{GRF}}^{g}$ acting on the foot are found by summing the contributions of each force sensor. The moment around the $x$ and $y$-axes can be represented using the CoP. The CoP denotes the point on the contact surface between the instrumented shoe and the ground, where the moments around the horizontal axes are zero. This means the moment measured by the force sensors, expressed in the global coordinate frame $\Psi_{g}$, can be described by: $\boldsymbol{M}_{\mathrm{GRF}}^{g}=\boldsymbol{x}_{\mathrm{CoP}}^{g} \times$
$\boldsymbol{F}_{\mathrm{GRF}}^{g}$. Solving this equation for $\boldsymbol{x}_{\mathrm{CoP}}^{g}$, and excluding the moment around the $z$-axis results in

$$
\boldsymbol{F}^{g}=\left(\begin{array}{c}
F_{x}^{g} \\
F_{y}^{g} \\
F_{z}^{g}
\end{array}\right) \quad \boldsymbol{x}_{C o P}^{g}=\left(\begin{array}{c}
-\frac{M_{y}^{g}}{F_{z}^{g}} \\
M_{x}^{g} \\
F_{z}^{g} \\
0
\end{array}\right) \quad \boldsymbol{M}^{g}=\left(\begin{array}{c}
0 \\
0 \\
M_{z}^{g}
\end{array}\right) .
$$

It should be noted that the $z$-component of the CoP is zero, since the $\mathrm{CoP}$ must be on the ground. Besides that, the subscipt $\mathrm{GRF}$ has been omitted for clarity.

\section{Three-Dimensional Foot Position and Orientation Estimation}

For the coordinate transformation from sensor to global coordinates, the transformation matrix $\boldsymbol{H}_{s}^{g}$ has to be calculated (Section II-A). This means the relative 3-D position and orientation of the sensors have to be estimated, which is achieved by combining the signals of an inertial sensor consisting of three accelerometers, and three rate gyroscopes. The estimation requires integration of the angular velocity $\boldsymbol{\omega}$, measured with the rate gyroscopes, to orientation, and double integration of the acceleration $a$, measured with the accelerometers, to position in global coordinates. The integration of the angular velocity $\boldsymbol{\omega}$ to orientation $\boldsymbol{R}_{s}^{g}$, is performed by solving the differential equation [24]

$$
\dot{\boldsymbol{R}}_{s}^{g}=\boldsymbol{R}_{s}^{g} \tilde{\boldsymbol{\omega}}_{s}^{s, g}
$$

where $\tilde{\boldsymbol{\omega}}_{s}^{s, g}$ is the skew-symmetric matrix of the angular velocity of frame $\Psi_{s}$ with respect to $\Psi_{g}$, expressed in $\Psi_{s}$

$$
\tilde{\omega}=\left(\begin{array}{ccc}
0 & -\omega_{z} & \omega_{y} \\
\omega_{z} & 0 & -\omega_{x} \\
-\omega_{y} & \omega_{x} & 0
\end{array}\right) .
$$

In general, position estimation is not possible without additional sources, due to the integration drift of the accelerometers. However, during walking certain initial and final conditions can be assumed [19] (e.g., zero velocity update [18], vertical position of foot equal at each step), and the integration time is limited (about one second). In addition, the relative position of a sensor touching the ground can be estimated from the orientation data separately, instead of straightforward integration of the accelerometer signals.

The algorithm for the relative 3-D foot position and orientation estimation of each stride is based on these principles, and shown in Fig. 3. The determination of the start and the end of a stride is based on force sensor information. The angular velocity $\boldsymbol{\omega}$ is integrated (9), and the initial and final conditions are used for drift compensation. The algorithm checks for heel down (HD) or heel off (HO), based on force sensor information. On the one hand, when the heel force sensor touches the ground (HD), the motion of the shoe is constrained, and the position determination is based on the orientation and knowledge about the distance between the force sensors under the shoe. During this phase, the absolute distance between the sensors 


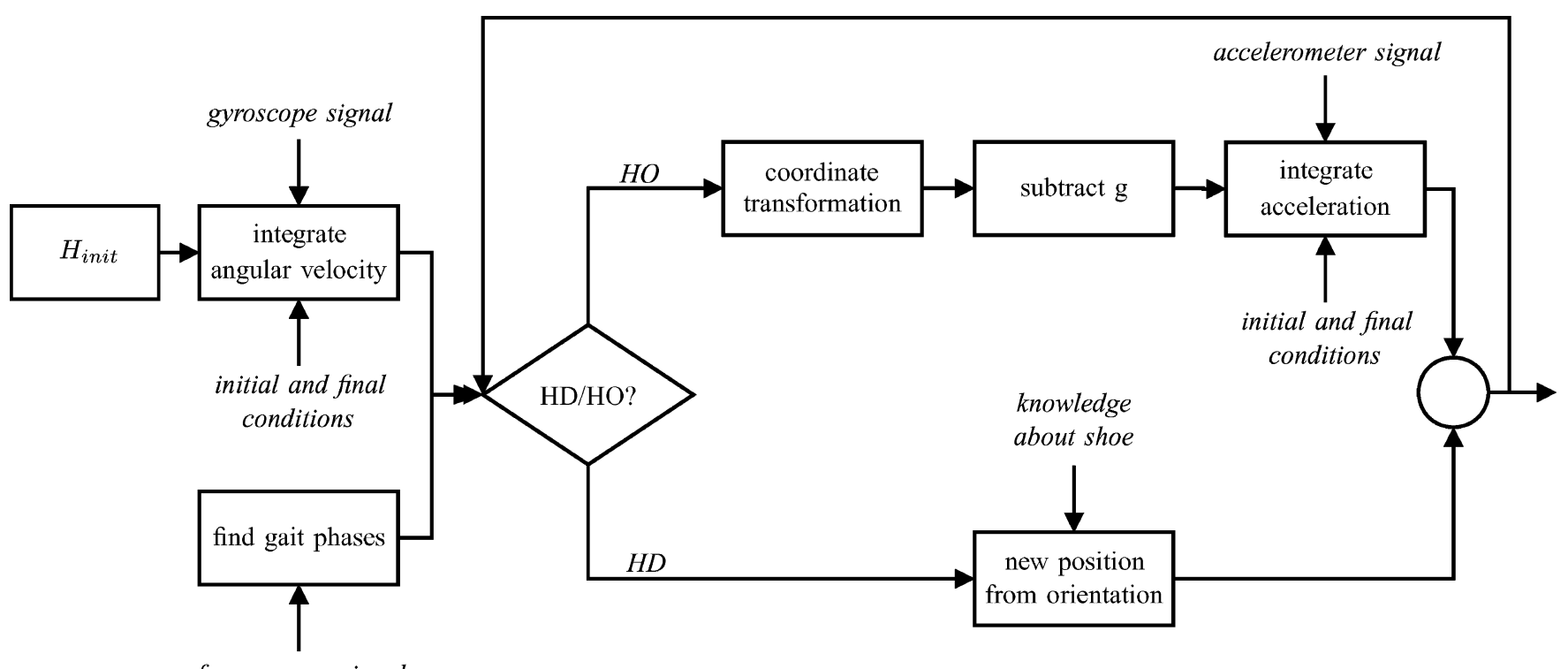

force sensor signal

Fig. 3. Flowchart of the integration algorithm to obtain position and orientation of a stride ( $H O=$ heel off, $H D=$ heel down).

is assumed to be constant, and the position of the sensors can be extracted from the last column of the transformation matrix (1). On the other hand, when the heel sensor does not touch the ground $(\mathrm{HO})$, the position is determined by straightforward integration of the accelerometer signal. First, the estimated orientation is used to transform the measured acceleration $\boldsymbol{s}$ from sensor coordinates to global coordinates, and the gravitational acceleration $\boldsymbol{g}$ is subtracted to result in the acceleration $\boldsymbol{a}$ of the sensor. Subsequently, to obtain the position of the sensors, this acceleration has to be integrated twice assuming the initial and final conditions to avoid drift, as shown by (11)

$$
\begin{aligned}
& \boldsymbol{v}^{-}(t)=\boldsymbol{v}_{0}+\int_{t_{s}}^{t} \boldsymbol{a}(\tau) d \tau \\
& \boldsymbol{v}^{+}(t)=\boldsymbol{v}^{-}(t)-\frac{t-t_{s}}{t_{e}-t_{s}} \boldsymbol{v}^{-}\left(t_{e}\right) \\
& \boldsymbol{x}^{-}(t)=x_{0}+\int_{t_{s}}^{t} \boldsymbol{v}(\tau) d \tau \\
& x_{z}^{+}(t)=x_{z}^{-}(t)-\frac{t-t_{s}}{t_{e}-t_{s}} x_{z}^{-}\left(t_{e}\right) ; \quad t=t_{s}, \ldots, t_{e}
\end{aligned}
$$

where $t_{s}, \ldots, t_{e}$ denotes the time interval when the heel sensor does not touch the ground. A minus superscript denotes the estimation of the signal before the drift compensation, whereas a plus superscript denotes the estimation of the signal after the drift compensation has been applied. It should be noted that the initial conditions $\boldsymbol{v}_{0}$ and $\boldsymbol{x}_{0}$ are zero, since the global coordinate system is renewed for each foot placement, as mentioned in Section II-A.

\section{Experimental Methods}

During the experiments, a healthy subject wearing instrumented shoes (total mass $68 \mathrm{~kg}$ ), was asked to walk repeatedly over an AMTI force plate. The instrumented shoes consisted of standard orthopaedic sandals equipped with two six degrees of freedom force/moment sensors (ATI-Mini45-SI-580-20, Schunk GmbH \& Co. KG) under the heel and forefoot, as shown in Fig. 1. Each sensor was enclosed between two aluminum mounting plates and carbon plates to assure rigidity and easy mounting. Furthermore, a thin piece of rubber provided friction between the lower carbon plates and the ground. The sole of the sandal between the sensors was not adapted, which resulted in a remaining flexibility at this part of the sole. Each force sensor had a miniature inertial sensor (Xsens Motion Technologies) attached to it, for the estimation of position and orientation. The position of the ankle was determined by assuming a fixed position in the heel segment, and using the information from the inertial sensors. To estimate the power at the ankle, the angular velocity at the ankle should be known. Since this angular velocity is given by the difference between the angular velocities of the heel and the lower leg, a third inertial sensor was attached to the lower leg to measure its angular velocity. The combination of an instrumented shoe and inertial sensors was compared to a reference measurement system consisting of an AMTI force plate and an optical position measurement system (Vicon, Oxford Metrics).

At the start of a measurement, the inertial sensors had to be calibrated with respect to the position and orientation of the force sensors. For the inertial sensors attached to the force sensors, this was simply done by measuring the distances between the inertial sensors and the force sensors, and knowing the initial orientation with respect to each other. For the inertial sensor on the lower leg, this was done by a sensor to segment calibration. The $z$ direction was determined by measuring the (gravitational) acceleration while keeping the lower leg in an upright position. The $y$-direction was determined by bending the knee. The $x$ direction was determined using the $y$ and $z$ direction, in order to obtain an orthogonal right-handed coordinate system. The position of the ankle in the heel segment was determined by measuring the distance between the ankle and the heel sensor. 

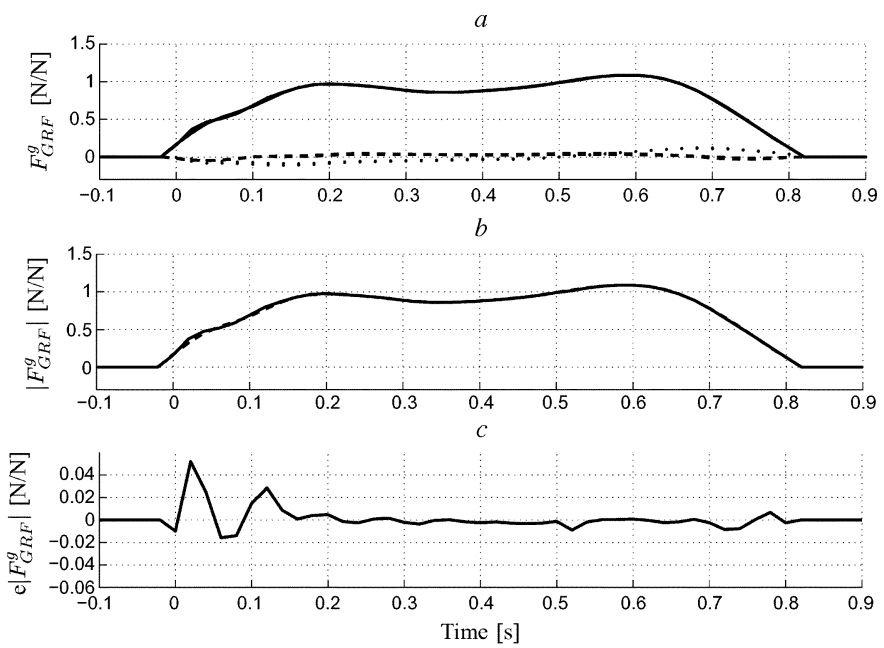

Fig. 4. GRF measured by instrumented shoe and force plate. (a) Components of GRF ( $x$ : dotted; $y$ : dashed; $z$ : solid). (b) Magnitude of complete GRF measured by the instrumented shoe (solid), and the force plate (dashed). (c) Error signal.

Each of the ten evaluated trials consisted of four strides, one of which was on the force plate. The analogue data from the force plate and force sensors were acquired at a sample rate of $1000 \mathrm{~Hz}$, the 3-D marker data at $50 \mathrm{~Hz}$, and the data from the inertial sensors at $50 \mathrm{~Hz}$. All data were low-pass filtered by applying a second-order Butterworth filter, both forward and reverse at a cutoff frequency of $15 \mathrm{~Hz}$. The synchronization between the inertial sensor system and Vicon was done by maximizing the correlation between the angular velocities of the lower leg estimated with both systems. Subsequently, all signals were resampled to a frequency of $50 \mathrm{~Hz}$, and possible gaps in the Vicon data were spline-interpolated prior to filtering. The voltages from the force sensors and the force plate were converted to forces by applying the calibration tables supplied by the manufacturer.

\section{RESULTS}

The three components of the measured GRF of a representative trial are shown in Fig. 4(a). It should be noted that the forces, moments, and powers are normalized with respect to body weight. The magnitude of the complete GRF is shown in Fig. 4(b), and the error between the magnitudes in Fig. 4(c). The signals show good correspondence, which is confirmed by Fig. 4(c), and the root mean square (RMS) difference over 10 evaluated trials of $(0.012 \pm 0.001) \mathrm{N} / \mathrm{N}$ (mean \pm standard deviation), being $(1.1 \pm 0.1) \%$ of the maximal GRF magnitude. The RMS difference of the estimates of the horizontal component of the GRF was $(0.017 \pm 0.008) \mathrm{N} / \mathrm{N}$, which corresponds to $(1.6$ $\pm 0.8) \%$ of the maximal GRF magnitude, or $(16 \pm 8) \%$ of the maximal horizontal component of the GRF. A separate analysis of each horizontal component results for the $x$ direction in an RMS difference of $(0.019 \pm 0.008) \mathrm{N} / \mathrm{N}$, being $(1.8 \pm 0.8) \%$ of the maximal GRF magnitude, or $(18 \pm 8) \%$ of the maximal $x$ component. For the $y$ direction in an RMS difference of $(0.007$ $\pm 0.002) \mathrm{N} / \mathrm{N}$, being $(0.7 \pm 0.2) \%$ of the maximal GRF magnitude, or $(15 \pm 5) \%$ of the maximal $y$ component. The estimation of the position of the CoP using the instrumented shoes, as well as the force plate is shown in Fig. 5. The trajectories agree

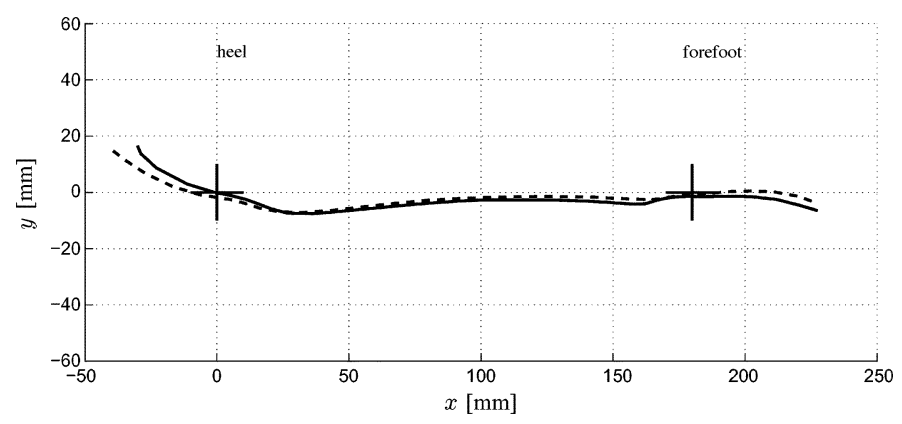

Fig. 5. CoP of instrumented shoe (solid) and force plate (dashed) expressed in global coordinate system. The center of each force sensor is indicated by the black cross.

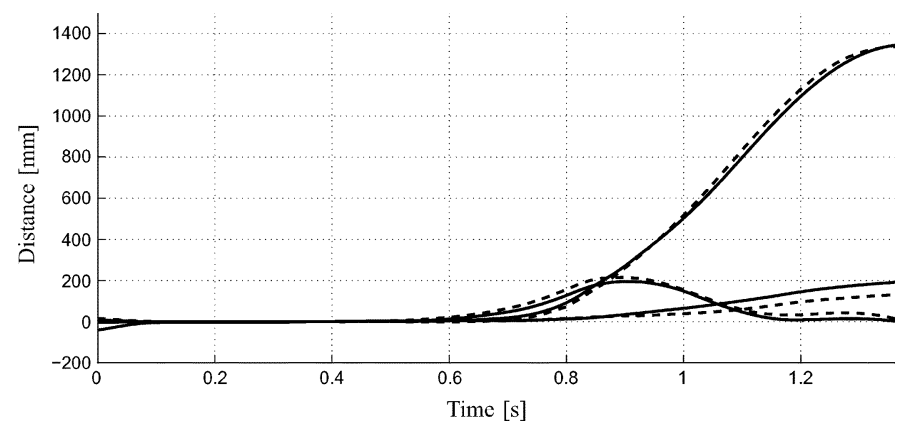

Fig. 6. Position of heel sensor estimated using instrumented shoes and inertial sensors (solid), and force plate and Vicon (dashed).

well, resulting in an RMS difference between both methods of $(5.1 \pm 0.7) \mathrm{mm}$, corresponding to $(1.7 \pm 0.3) \%$ of the length of the shoe. The estimation of the position of the heel force sensor using both methods is shown in Fig. 6. The RMS difference between the magnitudes of the position estimates using both methods was calculated as $(18 \pm 6) \mathrm{mm}$, being $(1.4 \pm 0.5) \%$ of the maximal magnitude. An integration of the estimated CoP, GRF, the position and orientation of the heel sensor is shown in Fig. 7. The figure indicates the possibility to measure several steps during a single measurement. It is easy to recognize the characteristic M-shape of the GRF, as shown in Fig. 4(b).

A comparison of the moment around the ankle is shown in Fig. 8. Fig. 8(a) shows the three components of the estimated ankle moment, using Vicon \& force plate as well as inertial sensors and instrumented shoes. The magnitude of the estimated ankle moment is shown in Fig. 8(b), and the error between the magnitudes in Fig. 8(c). The RMS difference over the 10 trials was $(0.004 \pm 0.001) \mathrm{Nm} / \mathrm{N}$, being $(2.3 \pm 0.5) \%$ of the maximal magnitude. The estimated power at the ankle is shown in Fig. 9(a). The RMS difference over the 10 evaluated trials was $(0.02 \pm 0.005) \mathrm{W} / \mathrm{N}$, being $(14 \pm 5) \%$ of the maximal power. Since the ankle moment shows good correspondence (Fig. 8), this relatively large difference is caused by an error in the estimation of the angular velocity (5), which is shown in Fig. 10. It should be noted that the $x$ and $z$ direction of the angular velocities are not shown, since their magnitude is small compared to the $y$ direction. Moreover, merely the difference between heel and lower leg angular velocity is shown for clarity. The RMS differences of the angular velocities are $(0.85 \pm 0.10)$ $\mathrm{rad} / \mathrm{s},(0.53 \pm 0.11) \mathrm{rad} / \mathrm{s}$, and $(0.89 \pm 0.07) \mathrm{rad} / \mathrm{s}$ for the heel, 


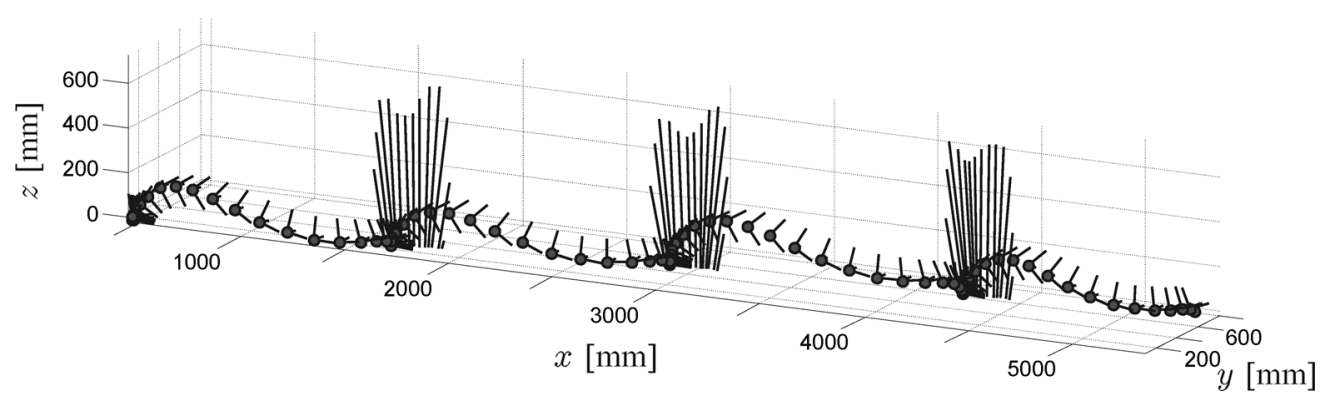

Fig. 7. CoP, GRF, position, and orientation of the heel sensor during several steps. The CoP and GRF of three steps are indicated by the lines pointing upwards. The position of the heel sensor is indicated by the dots, the corresponding orientation by the three lines representing the heel sensor coordinate axes.
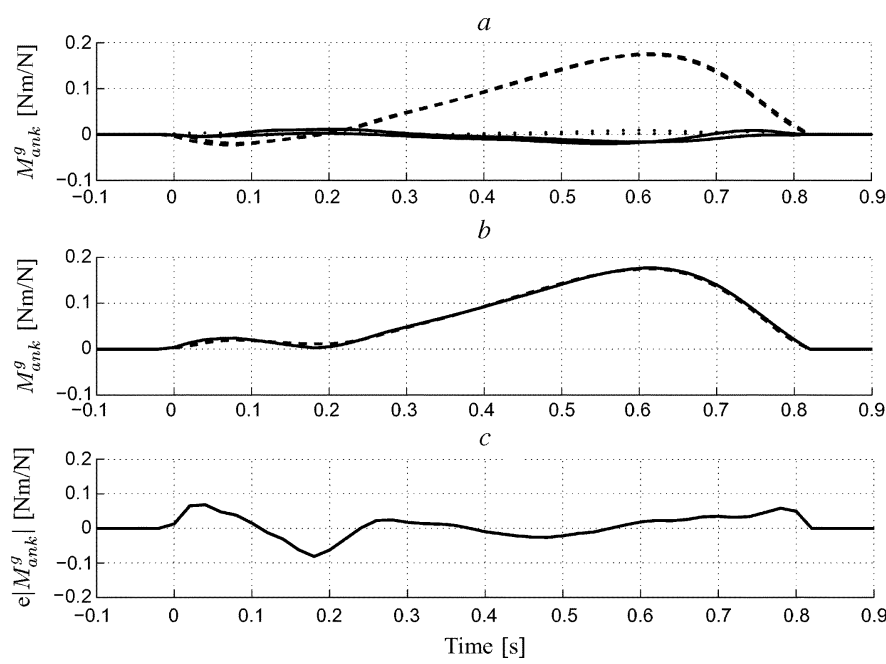

Fig. 8. Ankle moment estimated using instrumented shoes and inertial sensors, and force plate and Vicon. (a) Components of the ankle moment ( $x$ : dotted; $y$ : dashed; $z$ : solid). (b) Magnitude of the ankle moment measured by the instrumented shoe (solid), and the force plate (dashed). (c) Error signal.

lower leg, and the difference between them respectively, which corresponds to $(14 \pm 2) \%,(19 \pm 4) \%$, and $(19 \pm 3) \%$ of the maximal angular velocity. During push-off the ankle moment is maximal, which means a small error in the angular velocity during push-off gives rise to significant errors in the calculated ankle power, as illustrated by Fig. 9(a). Most probably, the angular velocity error is caused by an inaccurate estimation using Vicon for two reasons. First, the Vicon estimation requires a differentiation of marker position, whereas the inertial sensors measure the angular velocity directly using gyroscopes. The differentiation requires low-pass filtering to reduce the effect of high-frequency noise, which influences the results considerably. The effect is shown in Fig. 10, where the signal without filtering is rather noisy with a peak at toe off (dashed-dotted), whereas the low-pass filtered signal using a second-order Butterworth filter, both forward and reverse at a cutoff frequency of $5 \mathrm{~Hz}$ (dashed) results in leakage in the time domain. This leakage becomes clear at the start and the end of the stance phase, where the peaks are spread out over time. In contrast, the angular velocity measurement by the gyroscopes yields a smooth signal, while allowing a sufficient bandwidth to represent the angular velocity peak between heel and subsequent forefoot ground contact. Second, in the Vicon analysis the foot is modeled as one
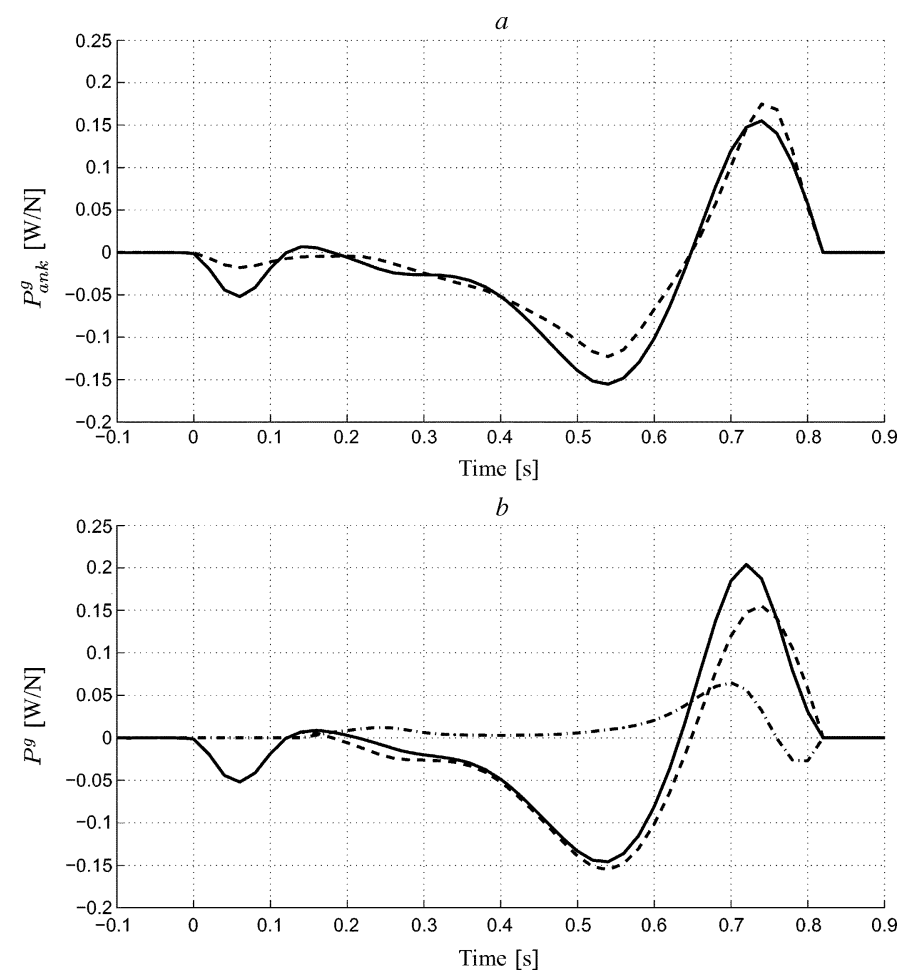

Fig. 9. Estimated power. (a) Ankle power using instrumented shoes and inertial sensors (dashed), and force plate and Vicon (solid). (b) Total power using instrumented shoes and inertial sensors (solid) calculated by the sum of ankle power (dashed), and foot power (dashed/dotted).

rigid segment, whereas this analysis uses two segments (Fig. 2). The wrong assumption of one rigid segment is confirmed by the relative change of distance between the markers positioned on the shoe, being $(7 \pm 1) \mathrm{mm}$ RMS during stance. It should be noted that the relative distance between the markers on the heel and the ankle changes as well [(7 \pm 1$) \mathrm{mm}$ RMS during stance], which is caused by deformation of the shoe cushioning, along with movement of the foot inside the shoe.

The relative movement between the two foot segments causes a foot moment and power as described by (6). The ankle power, the foot power, and their sum are shown in Fig. 9(b). For the calculation of the foot moment and power, an imaginary point of rotation was assumed at approximately the head of the first metatarsal bone. It should be noted that no reference is available for this signal, since the foot is assumed as one rigid segment in the Vicon analysis as is commonly done in such analysis [2]. 


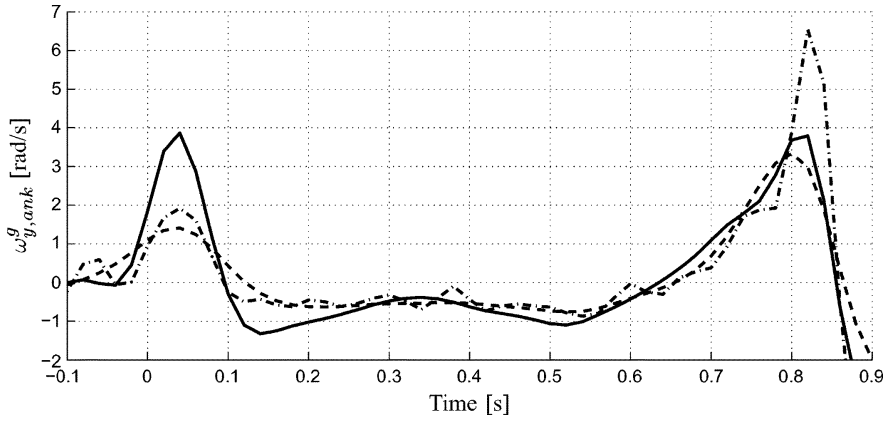

Fig. 10. Ankle angular velocity estimated using instrumented shoes and inertial sensors (solid), force plate and Vicon low-pass filtered using a second-order Butterworth filter, both forward and reverse at a cutoff frequency of $5 \mathrm{~Hz}$ (dashed), and force plate and Vicon without filtering (dashed/dotted).

\section{DISCUSSION}

Integration of body movement and GRF sensing, using the ambulatory setup proposed in the current study, yields a comprehensive analysis of ankle and foot dynamics including heel and forefoot movement, as well as ankle and foot moments and powers. The proposed measurement system is an extension of the system proposed by Veltink et al. [13]. Like Veltink's system, the GRF of a foot was measured using two six-degrees-of-freedom force sensors. In addition, the movement of foot and ankle was measured, using two inertial sensors. The angular velocity of the lower leg was measured by a third inertial sensor. The good reproduction of the ankle moment shows the feasibility of the measurement system for inverse dynamics applications problems.

However, the reproduction of the ankle power shows a rather large difference between the two evaluated methods. As described in Section III, the difference is caused by an inaccurate estimation of the angular velocities using Vicon. Standard marker configurations, as used in this research, do not allow the estimation of foot moment and power, since they do not separate the foot into multiple segments. In principle, more elaborate marker configurations could be used on the foot, which would allow foot moment and power to be estimated [25].

The biomechanical model of the foot used in our analysis consists of two segments (Fig. 2), whereas the reference model used in the Vicon analysis consists of one segment. For the two segments model, an imaginary point of rotation was assumed. In reality, however, the deformation of the foot and the shoe is distributed, and there is no single point of rotation. Therefore, the foot model should preferably include distributed deformation. The performance of the reference system can be improved by increasing the sample frequency, or by improving the accuracy of the position measurement, e.g., by using active markers, or by using more markers on the foot to measure the deformation of the foot. Principally, however, it is better to measure angular velocity directly, than to estimate it from position measurements by differentiation.

As mentioned in Section III, the relative distance between the markers on the heel and the ankle changes during stance. This means the assumption that the ankle position is fixed in the heel segment should be reconsidered. However, the estimated ankle moment shows good correspondence and, therefore, the assumption has not been reconsidered in this research.

The position and orientation estimates reported in this study have been achieved after considering initial and final conditions for a step. This results in a systematic delay which can not be compensated for by high computational power or efficient implementation of the algorithm. If the foot position and orientation will be computed realtime during a step, higher errors will occur because of integration drift, which can only be corrected after heel contact and application of the final conditions.

The measurements in this study have been performed in a gait laboratory. The subject walked in a straight line to compare several steps of a trial, and for an easy comparison with the force plate. However, the proposed system allows for ambulatory measurement over any number of consecutive steps including a change of direction while walking.

The design of the instrumented shoe can still be improved. Currently, the sensors are mounted between two aluminum plates, which are rather stiff. However, the sole beneath the sensor does not need to be very stiff for valid measurement of the GRF and, thus, some flexibility can be introduced. Moreover, the orientation of the force sensor is constantly measured by the inertial sensors, which allows the design optimization. Another possible optimization of the design would be a reduction of the weight of the sensors and mounting plates, which has not been optimized in the current design. It should be noted that the current design does have a small effect on the walking pattern, which is shown in an evaluation performed by Liedtke et al. [26]. In this study, gait on the instrumented shoe was compared to gait on normal, light, and heavy shoes. Gait parameters evaluated were stride length, stride width, maximum lateral foot excursion, stride time, stance time, and double stance time. However, a significant difference was only found in maximal GRF.

The proposed measurement system has been applied to a healthy subject. An important clinical application is the biomechanical analysis of patients having central neurological disorders, or lower leg amputees. It is, therefore, useful to see if it is possible to apply the proposed measurement system to those patients. Initial tests indicate that the shoes do not impede their walking.

\section{REFERENCES}

[1] D. A. Winter, Biomechanics and Motor Control of Human Movement, 2nd ed. New York: Wiley, 1990.

[2] H. F. J. M. Koopman, H. J. Grootenboer, and H. J. de Jongh, "An inverse dynamics model for the analysis, reconstruction and prediction of bipedal walking," J. Biomech., vol. 28, no. 11, pp. 1369-1376, 1995.

[3] A. Forner-Cordero, H. F. J. M. Koopman, and F. C. T. van der Helm, "Inverse dynamics calculations during gait with restricted ground reaction force information from pressure insoles," Gait Posture, vol. 23, no. 2, pp. 189-199, 2006.

[4] A. H. Hansen, D. S. Childress, S. C. Miff, S. A. Gard, and K. P. Mesplay, "The human ankle during walking: Implications for design of biomimetic ankle prostheses," J. Biomech., vol. 37, no. 10, pp. 1467-1474, 2004.

[5] M. Kljajić and J. Krajnik, "The use of ground reaction measuring shoes in gait evaluation," Clin. Phys. Physiol. Meas., vol. 8, pp. 133-142, 1987.

[6] S. Barnett, J. L. Cunningham, and S. West, "A comparison of vertical force and temporal parameters produced by an in-shoe pressure measuring system and a force platform," Clin. Biomech., vol. 15, no. 10, pp. 781-785, 2000. 
[7] K. J. Chesnin, L. Selby-Silverstein, and M. P. Besser, "Comparison of an in-shoe pressure measurement device to a force plate: Concurrent validity of center of pressure measurements," Gait Posture, vol. 12, pp. $128-133,2000$.

[8] A. Forner-Cordero, H. F. J. M. Koopman, and F. C. T. van der Helm, "Use of pressure insoles to calculate the complete ground reaction forces," J. Biomech., vol. 37, pp. 1427-1432, 2004.

[9] H. H. C. M. Savelberg and A. L. H. de Lange, "Assessment of the horizontal, fore-aft component of the ground reaction force from insole pressure patterns by using artificial neural networks," Clin. Biomech., vol. 14, pp. 585-592, 1999.

[10] M. A. Razian and M. G. Pepper, "Design, development, and characteristics of an in-shoe triaxial pressure measurement transducer utilizing a single element of piezoelectric copolymer film," IEEE Trans. Neural Syst. Rehabil. Eng., vol. 11, no. 3, pp. 288-293, Sep. 2003.

[11] E. S. Roland, M. L. Hull, and S. M. Stover, "Design and demonstration of a dynamometric horseshoe for measuring ground reaction loads of horses during racing conditions," J. Biomech., vol. 38, pp. 2102-2112, 2005.

[12] L.-P. Chao and C.-Y. Yin, "The six-component force sensor for measuring the loading of the feet in locomotion," Mater. Des., vol. 20, no. 5, pp. 237-244, 1999.

[13] P. H. Veltink, C. B. Liedtke, E. Droog, and H. van der Kooij, "Ambulatory measurement of ground reaction forces," IEEE Trans. Neural Syst. Rehabil. Eng., vol. 13, no. 3, pp. 423-427, Sep. 2005.

[14] D. Roetenberg, H. J. Luinge, C. T. M. Baten, and P. H. Veltink, "Compensation of magnetic disturbances improves inertial and magnetic sensing of human body segment orientation," IEEE Trans. Neural Syst. Rehabil. Eng., vol. 13, no. 3, pp. 395-405, Sep. 2005.

[15] D. Roetenberg, C. T. M. Baten, and P. H. Veltink, "Estimating body segment orientation by applying inertial and magnetic sensing near ferromagnetic materials," IEEE Trans. Neural Syst. Rehabil. Eng., 2006, to be published.

[16] H. J. Luinge and P. H. Veltink, "Measuring orientation of human body segments using miniature gyroscopes and accelerometers," Med. Biol. Eng. Comput., vol. 43, no. 2, pp. 273-282, 2005.

[17] E. R. Bachmann, "Inertial and magnetic tracking of limb segment orientation for inserting humans into synthetic environments," Ph.D. thesis, Naval Postgraduate School, Monterey, CA, 2000.

[18] R. K. Curey, M. E. Ash, L. O. Thielman, and C. H. Barker, "Proposed IEEE inertial systems terminology standard and other inertial sensor standards," presented at the IEEE Position Location Navigation Symp. 2004, Monterey, CA, 2004.

[19] P. H. Veltink, P. Slycke, J. Hemssems, R. Buschman, G. Bultstra, and H. Hermens, "Three dimensional inertial sensing of foot movements for automatic tuning of a two-channel implantable drop-foot stimulator," Med. Eng. Phys., vol. 25, pp. 21-28, 2003.

[20] D. Roetenberg, "Inertial and magnetic sensing of human motion," $\mathrm{Ph}$.D. thesis, Univ. Twente, Enschede,, The Netherlands, 2006.

[21] A. M. Sabatini, C. Martelloni, S. Scapellato, and F. Cavallo, "Assessment of walking features from foot inertial sensing," IEEE Trans. Biomed. Eng., vol. 52, no. 3, pp. 486-494, Mar. 2005.

[22] I. P. I. Pappas, M. R. Popovic, T. Keller, V. Dietz, and M. Morari, "A reliable gait phase detection system," IEEE Trans. Neural Syst. Rehabil. Eng., vol. 9, no. 2, pp. 113-125, Jun. 2001.

[23] A. L. Hof, "An explicit expression for the moment in multibody systems," J. Biomech., vol. 25, no. 10, pp. 1209-1211, 1992.

[24] J. E. Bortz, "A new mathematical formulation for strapdown inertial navigation," IEEE Trans. Aerosp. Electron. Syst., vol. 7, no. 1, pp. 61-66, Jan. 1971.

[25] B. A. MacWilliams, M. Cowley, and D. E. Nicholson, "Foot kinematics and kinetics during adolescent gait," Gait Posture, vol. 17, no. 3, pp. 214-224, 2003.
[26] C. B. Liedtke, S. A. W. Fokkenrood, J. T. Menger, H. van der Kooij, and P. H. Veltink, "Evaluation of instrumented shoes for ambulatory assessment of ground reaction forces," Gait Posture, 2006, to be published.

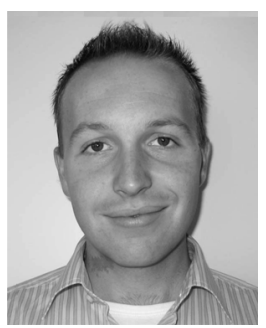

H. Martin Schepers ( $\left.\mathrm{S}^{\prime} 06\right)$ was born in Hardenberg, The Netherlands, on July 4, 1981. In 2004, he received the M.Sc. degree in electrical engineering from the University of Twente, Enschede, The Netherlands, where he is currently working toward the Ph.D. degree.

His field of research includes ambulatory sensor systems, movement analysis, and biomedical signal processing.

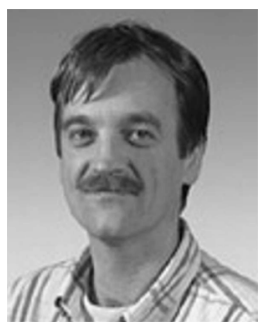

H. (Bart) F. J. M. Koopman received the M.Sc. degree in mechanical engineering with specialization fluid dynamics from the University of Twente, Enschede, The Netherlands. He performed his civil service at the Biomechanics Group of the University of Twente, which resulted in the Ph.D. degree at the end of 1989 on the analysis and prediction of human walking.

When the Laboratory of Biomechanical Engineering was founded in 1990, he started working there as a Faculty Member of the Faculty of Mechanical Engineering, University of Twente, where he now chairs the Biomechanical Engineering Group. He worked on various topics related to the co-ordination of movement and with applications in rehabilitation and orthopaedics. Currently, his main research interest is in biomechatronics. This area studies the control of human movement and the information exchange with mechanical aids. Within the Institute for Biomedical Technology (BMTI) he works closely with the Biomedical Signals and Systems Group of the Faculty of Electrical Engineering and the Roessingh Rehabilitation Center in Enschede.

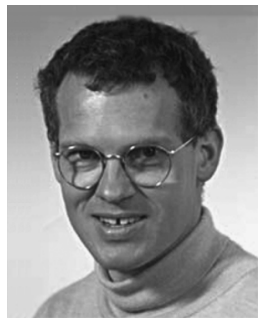

Peter H. Veltink (S'85-M'88-SM'06) was born in 1960 in Groenlo, the Netherlands. He received the M.Sc. (cum laude) in electrical engineering from the University of Twente, Enschede, The Netherlands, in 1984 and the Ph.D. degree in the area of electrical nerve stimulation, in 1988 .

Currently, he is a Professor of Technology for the Restoration of Human Function, the University of Twente, Institute for Biomedical Technology (BMTI), and performs research in the area of artificial motor control and ambulatory sensory systems with applications to rehabilitation medicine. He has been the scientific coordinator of $3 \mathrm{EU}$ research training networks and is and has been involved in various projects financed by the EU, the Dutch ministry of Economic Affairs, and the Dutch Foundation for Technical Sciences STW. He performed sabbaticals at Case Western Reserve University, Cleveland, OH, in 1989 and at the Center for Sensory-Motor-Interaction at Aalborg University, Aalborg, Denmark, in 1997.

Prof. Veltink has been the treasurer of the International Functional Electrical Stimulation Society (IFESS) from 1996 to 2001. He received the Royal Shell Stimulating Prize for his contribution to the rehabilitation-engineering field in 1997. 